\title{
Development of hip displacement in cerebral palsy: a longitudinal register study of 1,045 children
}

\author{
Philippe WAGNER ${ }^{1,3}$ and Gunnar HÄGGLUND ${ }^{1,2}$
}

\author{
${ }^{1}$ Department of Clinical Sciences, Lund University, Lund, Orthopedics; ${ }^{2}$ Department of Orthopedics, Skane University Hospital, Lund; 3 Centre for Clinical \\ Research, Uppsala University, Region Västmanland, Västerås, Sweden \\ Correspondence: philippe.wagner@regionvastmanland.se \\ Submitted 2021-06-25. Accepted 2021-10-11.
}

Background and purpose - Hip surveillance in children with cerebral palsy $(\mathrm{CP})$ includes repeated radiographic hip examinations and measurements of the hip migration percentage (MP) to identify hips in need of surgery early, to prevent dislocation with the fewest number of radiographic examinations possible. We analyzed the early development of the MP in hips operated on to prevent hip dislocation and hips stabilized without surgery

Patients and methods - From the Swedish Surveillance Programme for CP, 5,899 radiographic measurements from 1,045 children with a Gross Motor Function Classification System level III-V born in 1996-2011 were analyzed. For children operated on to prevent hip dislocation, measurements up to the most recent preoperative radiograph were included. The hip with highest MP was analyzed for each child. A mixed-effects model was used to estimate the development of the MP at each age for each child and the population mean.

Results - In the 702 children who did not undergo preventive surgery, the mean MP increased with decreasing velocity up to age 6 years. Here it reached $24 \%$ (95\% confidence interval [CI] 24-25), with a velocity of $0.3 \%$ year (CI $0.0-0.5$ ), remaining approximately stable up to age 12 years. In the 343 children who underwent preventive surgery (219 adductor-psoas lengthening, 124 varus derotation osteotomy of proximal femur), the mean MP increased with an increasing velocity from a mean of 30\% (CI 27-32) 3 years before the operation.

Interpretation - An increasing rate of hip displacement in hips with an MP $>24 \%$ indicates the need for preventive surgery. Hips stabilized without preventive surgery had a decreasing displacement rate and were usually stabilized with an MP $<30 \%$ at age 6 years.
Children with cerebral palsy (CP) are at risk of developing hip dislocation because of altered muscle forces across the hip joint (1). The lateral displacement of the femoral head develops gradually, which means that it can be identified and treated before complete dislocation occurs if the hips are followed up with repeated radiographic examinations $(2,3)$.

The degree of lateral displacement of the femoral head is usually measured with the Reimers migration percentage (MP; Figure 1). An MP $>30-33 \%$ is often regarded as a hip displacement and an MP > 90-100\% as hip dislocation (4). Several hip surveillance programs have been described $(3,5,6)$. Most programs base the radiographic follow-up schedule on the child's age, MP, and degree of motor impairment classified according to the Gross Motor Function Classification System (GMFCS) (7). The recommended frequency of radiographic examinations at different ages and GMFCS levels varies between these programs.

The Swedish Surveillance Program for CP (CPUP) is a follow-up program and register for individuals with CP that started in southern Sweden in 1994 (1.2 million inhabitants) and since 2006 has included $>95 \%$ of all children and ado-

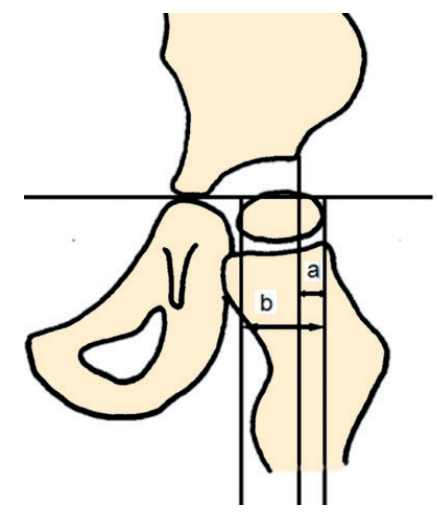

Figure 1. Reimers migration percentage $(M P)=a / b \times 100$. 
lescents with CP in Sweden (10.4 million inhabitants) born in 2000 and later (8). In CPUP, hips are followed up with repeated measurements of MP on anteroposterior pelvic radiographs.

We analyzed and compared the early development of the MP in hips operated on with preventive surgery (adductorpsoas lengthening [APL] or varus derotation osteotomy of the proximal femur [VDRO]) and hips not operated on with preventive surgery in the population of children with CP enrolled in CPUP.

\section{Patients and methods}

In Sweden, children with suspected CP are offered participation in CPUP as early as possible. After the age of 4 years, the diagnosis of CP is confirmed by a neuropediatrician and children who do not have CP leave the program (8). The child's physiotherapist classifies the GMFCS level from lowest (level I) to greatest functional impairment (level V).

In CPUP, a pelvic anteroposterior view is used to examine children in GMFCS levels III-V once a year until 8 years of age and at 2 and 6 years of age in those in level II. Children in level I are not examined radiographically provided the child has a normal pain-free range of hip motion. After 8 years of age, the children are followed radiographically at individual intervals based on the results of the previous radiographic and clinical reports. Children without pelvic obliquity and an MP $<40 \%$ are followed until skeletal maturity, and those with an MP $>40 \%$ or pelvic obliquity are followed in adulthood.

In this study, we analyzed the reported measurements for the MP until December 31, 2017, obtained from pelvic radiographs for all children in GMFCS levels III-V born in 19962011. In children operated on to prevent hip dislocation, the measurements up to the most recent preoperative radiograph were included. Children on a waiting list for hip surgery and children with an MP $>70 \%$ for whom surgery was indicated, but the child's condition was deemed too poor for surgery, or whose parents refused operation, were excluded. To simplify the data analysis and presentation, measurements at each time point were summarized by comparing the right and left sides and classifying the worst and best hip in terms of the MP. Because treatment decisions are based on the worst hip, this was the focus of analysis in this study.

\section{Statistics}

\section{Outcome validation}

Stability of the outcome over time is examined in the Appendix (see Supplementary data).

\section{Description of measurements}

To describe the distribution of MP with age, 2 scatter plots were generated: 1 for children who underwent preventive hip surgery during the follow-up and 1 for children who did not. Population means by age, for the former, and by time to sur- gery, for the latter, stratified according to the best and worst sides, were estimated using linear regression and cubic splines in R (9) and added to the graphs. Means according to age were added as a crude description of the central tendency of the data for the best and worst sides.

\section{Analyses of trends in population and individual means with age}

Subsequent analyses were performed using the hip with highest MP for operated and nonoperated sides in separate strata. To account properly for the longitudinal structure of the study data we used mixed-effects models (MM) to estimate and analyze the change in the mean MP with time, for each child (10). The MM allowed us to account, at least in part, for systematic inclusion and dropouts (11) and to quantify individual developmental heterogeneity. The MM included a polynomial for age for the nonoperated strata, and for time to surgery for the operated strata, with both random and fixed effects for each coefficient. The degree of polynomials was determined using the Akaike information criterion (10).

We performed several analyses using these MMs. First, a graph showing the predicted population mean MP development with age and the associated $95 \%$ confidence interval (CI) for the nonoperated group was produced. Pointwise intervals containing all individual means within 1 standard deviation (SD) of the population mean were then added as calculated from the model random effects distribution as a measure of the heterogeneity of the individual means. An analogue curve was generated to illustrate the instantaneous MP velocity at each age together with $95 \%$ CIs and 1 SD intervals containing the individual means. For the operated group we used the time before surgery as the primary time axis. A velocity of less than $0.5 \%$ year was not considered clinically important, and hips moving at this pace were deemed sufficiently stable.

Residual plots were used to assess the fit of each model. As an additional MM validation, we ensured that the SD of the residual error was close to the reported measurement error of the MP measurements. To evaluate the potential bias because of the late inclusion of some children we used sensitivity analyses where all MM analyses were additionally performed by including only those starting the follow-up before the age of 3 years and who were subsequently followed for 3 years.

\section{Age-adjusted differences in trends}

To offset the development of MP before surgery against the development by age in the nonoperated group, we performed an analysis of adjusting for the current age at the follow-up. This was done by subtracting from each measurement at a specific age in the operated strata, the predicted population mean of the nonoperated strata at that same age. The resulting outcome was then analyzed using an MM, which produced an age-adjusted MP for the 3 years preceding and up to the time of surgery. 
Number of children (\%) included according to sex, GMFCS level, and preventive surgery performed

\begin{tabular}{lcrrr}
\hline Factor & \multicolumn{3}{c}{ GMFCS level } & \\
& III & V & Total \\
\hline Boys & $137(22.2)$ & $236(38.2)$ & $244(39.5)$ & 617 \\
Girls & $88(20.6)$ & $172(40.2)$ & $168(39.2)$ & 428 \\
No operation & $198(28.2)$ & $295(42.0)$ & $209(29.8)$ & 702 \\
APL & $20(9.1)$ & $74(33.8)$ & $125(57.1)$ & 219 \\
VDRO & $7(5.6)$ & $39(31.4)$ & $78(62.9)$ & 124 \\
Total & $225(21.5)$ & $408(39.0)$ & $412(39.4)$ & 1,045
\end{tabular}

GMFCS $=$ Gross Motor Function Classification System . $\mathrm{APL}=$ Adductor-psoas lengthening.

VDRO = Varus derotation osteotomy of proximal femur.

Analyses of factors affecting trends in development

Interaction terms were included in the MM to analyze the influence of GMFCS level, age at the time of surgery, and type of surgery performed (APL or VDRO) on the MP level before surgery and the MP development with age for the nonoperated hips. Using the maximum likelihood approach, we estimated 2 models (10) and tested them against each other using a likelihood ratio test: 1 model contained the polynomial only and the other contained additional interaction terms between each polynomial term and each of the given factors. All tests were 2 -sided, and p-values $<0.05$ were considered to be statistically significant. CI refers to the $95 \%$ confidence interval. All analyses were performed and graphs generated using R (12).

\section{Ethics, funding, and potential conflicts of interest}

The study was approved by the Medical Research Ethics Committee at Lund University (LU-443-99). The study was

funded by Stiftelsen för bistånd åt rörelsehindrade i Skåne. The authors declare no conflict of interest.

\section{Results}

1,076 children in GMFCS levels III-V were reported during the study period. Surgery was indicated in 31 children based on the radiographic appearance but was not performed because the child was on waiting list for surgery, due to parents' decision to refrain surgery, or because the child's health was considered to be too poor. The analyses were based on the remaining 1,045 children (617 boys, 428 girls) and included 5,899 pelvic radiographs (Table).

\section{Description of measurements}

In the 702 nonoperated children, the MP population mean increased up to 6 years of age in both hips, and the population mean remained stable at about $24 \%$ in the most displaced hip and $18 \%$ in the contralateral hip (Figure 2). In the 343 children who had received preventive surgery, the MP population mean increased during the 3 years preceding the operation (Figure $3)$. The 1st surgery was performed at a mean age of 5.8 years (0.9-12.0). The mean MPs at the time of surgery were estimated at $56 \%$ in the most displaced hip and $31 \%$ in the contralateral hip (Figure 3).

\section{Analyses of trends in development}

The MM for nonoperated children best described the data using a 4th-degree polynomial of age (Figure 4) and for operated children a 2nd-degree polynomial of time before surgery (Figure 5).

For the nonoperated children, a substantial increase in mean MP occurred at an early age and

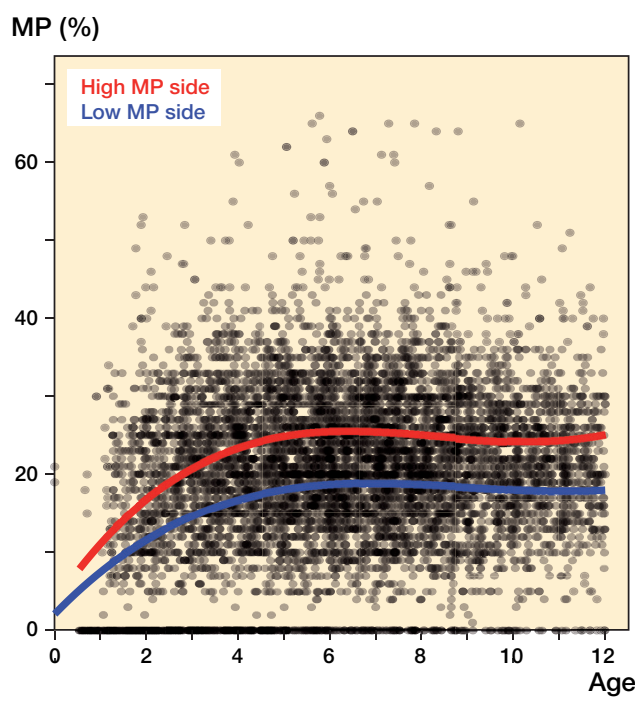

Figure 2. Hip displacement measured as the migration percentage (MP) in 702 children with CP in GMFCS levels III-V who did not undergo preventive hip surgery related to age.

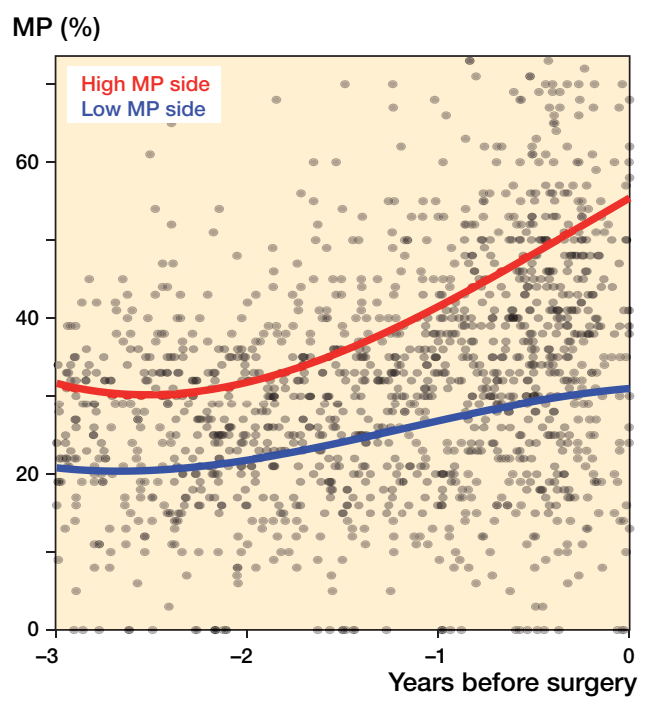

Figure 3. Hip displacement measured as the migration percentage (MP) in 343 children with $\mathrm{CP}$ in GMFCS levels III-V who underwent preventive hip surgery displayed according to the time before surgery. a high but decreasing velocity and a point of sufficient stability was reached at about 6 years of age. At age 1 year, the mean MP was $8.4 \%$ (CI 6.4-10) and MP velocity 9.7\%/year (CI 8.1-11). The corresponding values were $24 \%$ (CI 24-25) and $0.3 \% /$ year (CI $0.0-0.5)$ at age 6 years and $23 \%$ (95\% CI 22-24) and $-2.8 \%$ year (CI -4.2 to -1.3 ) at age 12 years (Figure 4 ). Sensitivity analysis indicated that the slight downturn toward the end of the follow-up may have reflected the influence of children included at a late age (data not shown).

For the operated children, the mean MP 3 years before surgery was 30\% (CI 27-32) and MP 


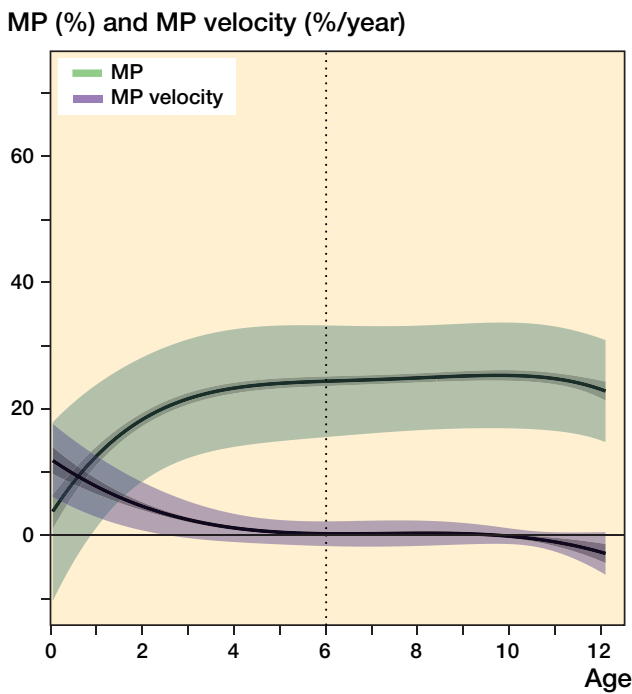

Figure 4. Model describing the development of population mean MP and MP velocity in the 702 children who did not undergo preventive hip surgery. 95\% Cls (dark) and 1 SD of individual variation (light) are shown. Vertical line shows point of $\mathrm{MP}$ velocity $<0.5 \% /$ year with $95 \% \mathrm{Cl}$.
MP (\%) and MP velocity (\%/year)

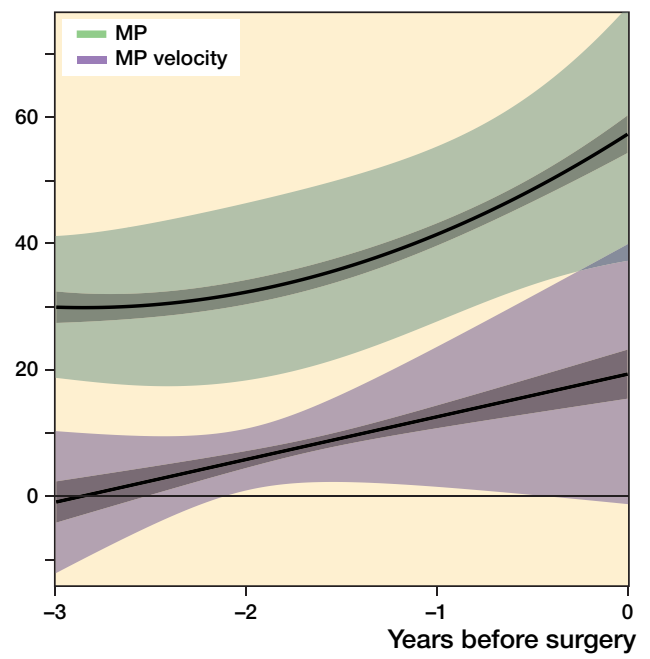

Figure 5. Model describing the development of population mean MP and MP velocity in the 343 children who underwent preventive hip surgery. $95 \%$ Cls (dark ) and 1 SD of individual variation (light) are shown. showed an increasing rate of displacement during the 3 years preceding the operation. The trends in development before surgery were similar regardless of the GMFCS level.

The primary cause of hip displacement in $\mathrm{CP}$ is the abnormal forces on the hip joint, which lead to the typical spastic position of the hip in adduction, flexion, and inward rotation. The spastic position changes the force direction from the superomedial to the superoposterolateral part of the acetabulum (1). The passive range of hip abduction often decreases with age in children with $\mathrm{CP}$ (13), which suggests that the increasing spastic position may explain the accelerating hip displacement found in hips in need velocity was $-1 \% /$ year (CI -4.1 to 2.4 ). At the time of surgery, the mean MP was 57\% (CI 54-60) and MP velocity was 19\%/ year (CI 15-23) (Figure 5).

The variation in individual development stratified by surgical status at the end of the follow-up is shown in the Figures 6 and 7 (see Supplementary data). The proportion of patients having had their 1st surgery at age $0-12$ years is shown in the Figure 8 (see Supplementary data). Graphs showing ageadjusted trends are shown in the Figures 9 and 10 (see Supplementary data).

\section{Factors affecting trends in development before the operation}

Of the factors examined, only the type of operation $(\mathrm{p}=0.01)$ and age at the time of the operation $(p<0.001)$ were significantly associated with MP development. The average MP on the most displaced side was 5.4\% lower (CI 0.4-10.5) in children who underwent APL compared with those who underwent VDRO. The MP on the most displaced side at the time of the operation was also $2.0 \%$ lower (CI 1.2-2.8) for each year of age at the time of the operation. The effect of the GMFCS level, the third factor examined, was small and not statistically significant $(\mathrm{p}=0.9)$.

\section{Discussion}

The main findings were that hips in children who did not undergo preventive surgery had a decreasing MP progression rate up to 6 years of age and were then stable at a population average of $24 \%$. Hips operated on with preventive surgery of preventive surgery.

From a methodological standpoint, the analysis of mean MP development with age is complicated by several factors.

$1 \mathrm{st}$, because the recommended indication for surgery is an MP > 40-50\% $(\mathbf{1 4 , 1 5})$, it follows by definition that most hips in the nonsurgical strata must stabilize below this level. Therefore, it is not possible to determine whether some children could stabilize at a hip MP $>40 \%$. However, our results appear to indicate that the MP development stabilizes at a much lower level for most children and that this is likely to be unaffected by the current indication for surgery.

A 2nd possible complication is that the time under study for each participant, from inclusion to the last MP measurement or time of surgery, which determined the number of measurements for each child, may have been affected by factors such as the way the surveillance program is implemented at each healthcare provider, the handling of patient queues and/or changes to the treatment guidelines, and variation in indications for surgery. This may have affected our ability to estimate individual trends as well as the MP level reached at the time of surgery. However, sensitivity analyses of how the results varied between birth cohorts and healthcare regions did not reveal substantial differences (data not shown), which leads us to conclude that the effects of these factors may be minor. Lastly, the follow-up at younger ages may be influenced by misclassification because some children are yet to have surgery. According to the inclusion criteria used, the youngest children were born in 2011 and would have reached age 7 years by the end of the study, whereas a large proportion of children had already had their 1st operation, as shown in the Figure 8 (see Supplementary data). 
In terms of statistical calculations, because the adjusted mean MP development curves were calculated by simply subtracting the predicted age-specific mean (ASM) from nonoperated children from each MP measurement of children operated on, the age-adjusted development CIs do not account for the sampling variation of the nonoperated group, i.e., the random variation in ASM that would occur if the study had been repeated. Instead, the CIs reflect a somewhat different study design where new data would have been collected on another group of operated children and adjusted using the ASM values of the present study. Crude calculations using standard errors from both groups, as well as additional clustered bootstrap analyses, indicate that CIs would increase by around $5-10 \%$ if the sampling variation of the nonoperated group was accounted for. Due to the complexity of the statistical analysis needed to properly account for this variation and the fact that results from the analysis are not part of the main focus of the study we instead performed this cruder analysis. There is uncertainty when measuring the MP relating to the quality of the radiograph and the examiner's experience. Parrott et al. (16) demonstrated that an experienced rater is expected to measure MP within 5.8\% of the true value. Faraj et al. (17) analyzed the error in the measurement of the MP with less experienced observers and reported a median intraobserver difference of $3.2-3.6 \%$ and median interobserver difference of 3.3-5\%. Our study is based on radiographs from many different departments in Sweden whose images were measured by the local orthopedic surgeon or radiologist, which reflects the situation in the CPUP surveillance program. When using radiographs to decide whether preventive surgery should be recommended, it is important to consider all the child's radiographs and the quality and possible uncertainty due to less-than-optimal positioning. It is also important to look at other factors, such as the head shaft angle (18) acetabular index, pelvic obliquity, age, and GMFCS level.

Most hips with an MP > 40-50\% need surgery to prevent dislocation $(14,15,19)$. It is an advantage to operate early before the femoral head becomes deformed and acetabular dysplasia necessitates a pelvic osteotomy. To avoid unnecessary operations, it is important to be able to predict which hips will require surgery with as high sensitivity and specificity as possible. Young age, high GMFCS level, high MP, and large head shaft angle are known risk factors for hip displacement $(20,21)$. The CPUP Hip Score calculates the risk of hip displacement in a hip with an MP $>40 \%$ based on these 4 risk factors and has an area under the ROC curve value of 0.87 (21). The current study shows that a high rate of displacement in hips with MP> $24 \%$ also indicates the need for preventive surgery.

Hip displacement often starts at an early age, which shows the importance of including children in a hip surveillance program as soon as possible. In CPUP, children are examined radiographically once a year until the age of 8 years and then individually based on previous surveys, usually every 2 nd year. The Australian and American Academy for Cerebral
Palsy and Developmental Medicine (AACPDM) programs recommend radiographic examinations 1-2 times per year up to age 7-8 years and thereafter annually until skeletal maturity for all children in GMFCS level V and, in the AACPDM program, also for those in GMFCS IV. More frequent radiographic examination before 8 years of age provides faster and more accurate information regarding MP development but involves a higher radiation dose. Based on our results, it seems possible to extend the intervals for children with a decreasing progression rate and stabilization at 8 years of age with no pelvic obliquity.

Our study has some limitations. Given the wide range of ages at inclusion and into the follow-up program, some uncertainty remains as to whether the trends shown here reflect the longitudinal development in the nonoperated population or late inclusion of children with a lower MP. However, the sensitivity analysis of those included early showed that the small decrease in MP after age 9-10 years may be related in part to this issue because this population appeared to stabilize with an MP of 20-25\% around age 6 years without the same slow decrease observed in the older children. Another limitation is that all children were not followed until skeletal maturity. However, we saw that stabilization had taken place and that most of the operations were performed before the age of 10 years. The 31 children with hips that were judged to need surgery, but for various reasons were not operated on, were excluded as they were regarded to represent an intermediate group. As they accounted for only $3 \%$ of the study cohort, this should not affect the results. A strength of our study is its prospective design based on the total population of children in Sweden in GMFCS levels III-V.

In conclusion, we found that hips that underwent preventive surgery showed an increasing displacement rate during the 3 years preceding the operation, whereas nonoperated hips showed a decreasing rate and usually stabilized with an MP of about $24 \%$ at age 6 years. Because hip displacement often occurs early, it is important to involve children in a hip surveillance program as early as possible.

Study design: GH, PW. Data collection: GH, PW. Statistical analysis: PW. Manuscript preparation: GH, PW.

Acta thanks Darko Anticevic and Line Kjeldgaard Pedersen for help with peer review of this study.

1. Miller F, Slomczykowski M, Cope R, Lipton G E. Computer modeling of the pathomechanics of spastic hip dislocation in children. J Pediatr Orthop 1999; 19: 486-92.

2. Dobson F, Boyd R N, Parrott J, Nattrass G R, Graham H K. Hip surveillance in children with cerebral palsy: impact on the surgical management of spastic hip disease. J Bone Joint Surg Br 2002; 84: 720-6.

3. Hägglund G, Andersson S, Düppe H, Lauge-Pedersen H, Nordmark E, Westbom L. Prevention of hip dislocation in children with cerebral palsy: the first ten years' experience of a population-based prevention programme. J Bone Joint Surg 2005; 87-B: 95-101. 
4. Reimers J. The stability of the hip in children: a radiological study of the results of muscle surgery in cerebral palsy. Acta Orthop Scand 1980; 184(Suppl.): 1-100.

5. Wynter M, Gibson N, Willoughby $\mathbf{K}$ L, Love S, Kentish M, Thomason P, et al. Australian hip surveillance guidelines for children with cerebral palsy:5-year review. Dev Med Child Neurol 2015; 57: 808-20.

6. Shrader M W, Wemberly L, Thompson R. Hip surveillance in children with cerebral palsy. J Am Acad Orthop Surg 2019; 27: 760-8.

7. Palisano R, Rosenbaum P, Walter S, Russell D, Wood E, Galuppi B. Development and reliability of a system to classify gross motor function in children with cerebral palsy. Dev Med Child Neurol 1997; 39: 214-23.

8. Alriksson-Schmidt A I, Arner M, Westbom L, Krumlinde-Sundholm L, Nordmark E, Rodby-Bousquet E, et al. A combined surveillance program and quality register improves management of childhood disability. Disabil Rehabil 2017; 39: 830-6.

9. Wickham H. ggplot2: elegant graphics for data analysis. New York: Springer Verlag; 2016. ISBN 978-3-319-24277-4. https://ggplot2.tidyverse.org

10. Fitzmaurice G M, Laird N M, Ware $\mathbf{J} \mathbf{H}$. Applied longitudinal analysis, 2nd ed. Chichester: Wiley; 2011. ISBN: 978-0-470-38027-7.

11. Mallinckrodt C H, Clark W S, David S R. Accounting for dropout bias using mixed-effects models. J Biopharm Stat 2001; 11: 9-21.

12. R Core Team. R: A language and environment for statistical computing. R Foundation for Statistical Computing, Vienna, Austria; 2018. https:// www.R-project.org/
13. Nordmark E, Hägglund G, Lauge-Pedersen $\mathbf{H}$, Wagner $\mathbf{P}$, Westbom L. Development of lower limb range of motion from early childhood to adolescence in cerebral palsy: a population-based study. BMC Med 2009; 7: 65 .

14. Shore B J, Yu X, Desai S, Selber P, Wolfe R, Graham H K. Adductor surgery to prevent hip displacement in children with cerebral palsy: the predictive role of the Gross Motor Function Classification System. J Bone Joint Surg Am 2012; 94: 326-34.

15. Terjesen T. To what extent can soft-tissue releases improve hip displacement in cerebral palsy? Acta Orthop 2017; 88: 695-700.

16. Parrott J, Boyd R N, Dobson F, Lancaster A, Love S, Oates J, et al. Hip displacement in spastic cerebral palsy: repeatability of radiologic measurement. J Pediatr Orthop 2002; 22: 660-7.

17. Faraj S, Atherton W G, Stott N S. Inter- and intra-measurer error in the measurement of Reimers' hip migration percentage. J Bone Joint Surg Br 2004; 86: 434-7.

18. Hermanson M, Hägglund G, Riad J, Wagner P. Head-shaft angle as risk factor for hip displacement in children with cerebral palsy. Acta Orthop 2015; 86: 229-32.

19. Hägglund G, Lauge-Pedersen H, Persson M. Radiographic threshold values for hip screening in cerebral palsy. J Child Orthop 2007; 1: 43-7.

20. Hägglund G, Lauge-Pedersen $\mathbf{H}$, Wagner $\mathbf{P}$. Characteristics of children with hip displacement in cerebral palsy. BMC Musculoskelet Disord 2007; 8: 101

21. Hermanson M, Hägglund G, Riad J, Rodby-Bousquet E, Wagner P. Prediction of hip displacement in children with cerebral palsy: development of the CPUP hip score. Bone Joint J 2015; 97-B: 1441-4. 


\section{Supplementary data}

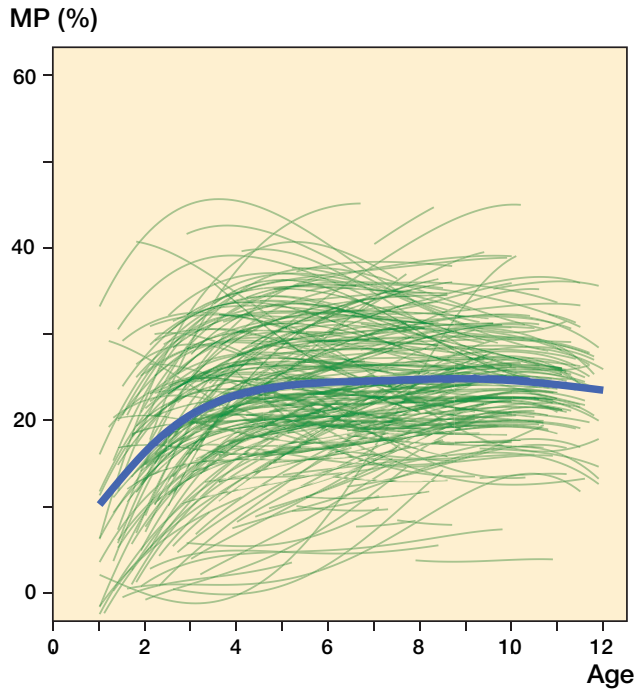

Figure 6. Predicted individual mean MP development in a random sample of 300 out of the 702 children who did not undergo preventive surgery together with the population mean.

\section{Appendix: Outcome validation}

To examine whether the definition of the most displaced side remains the same over time, we examined the mean number of times the side with the highest MP changed for each child. We quantified the difference between the right and left sides at each time the MP changed.

\section{Results}

The median number of times the side with the highest MP changed from the preceding occasion for each child was 2 . About $90 \%$ of times the side had changed from the preceding occasion, and the difference between sides was MP $\leq 10 \%$.

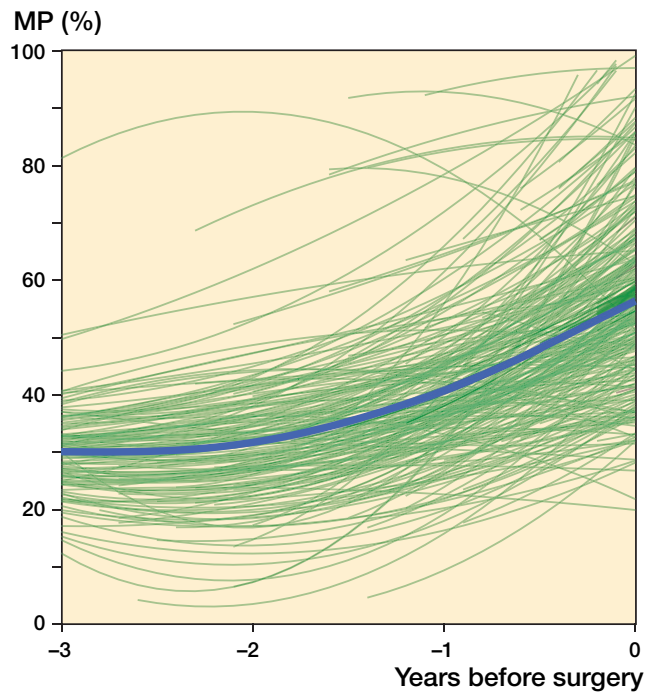

Figure 7. Predicted individual mean MP development in the 343 children who underwent preventive surgery together with the population mean.

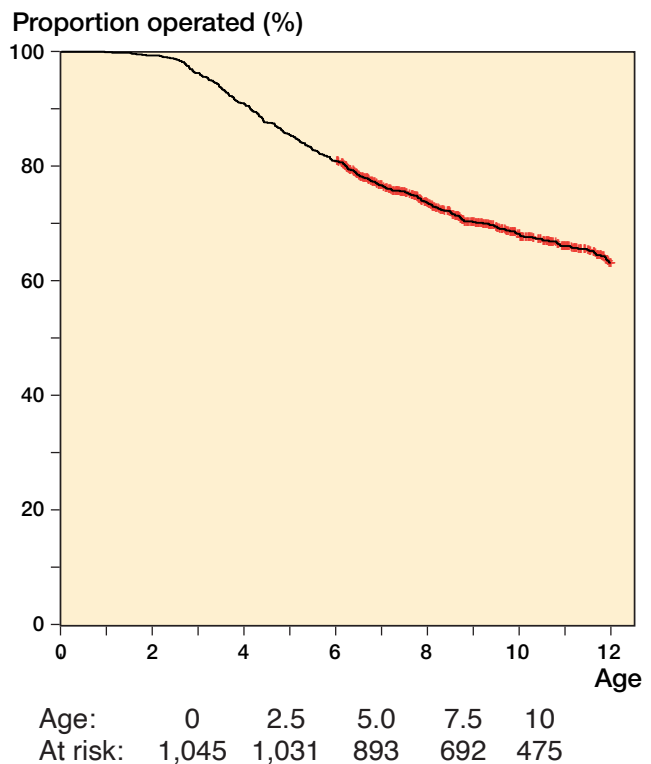

Figure 8. Predicted proportion of children having the 1 st surgery at different ages during the follow-up. 
Adjusted MP (\%)

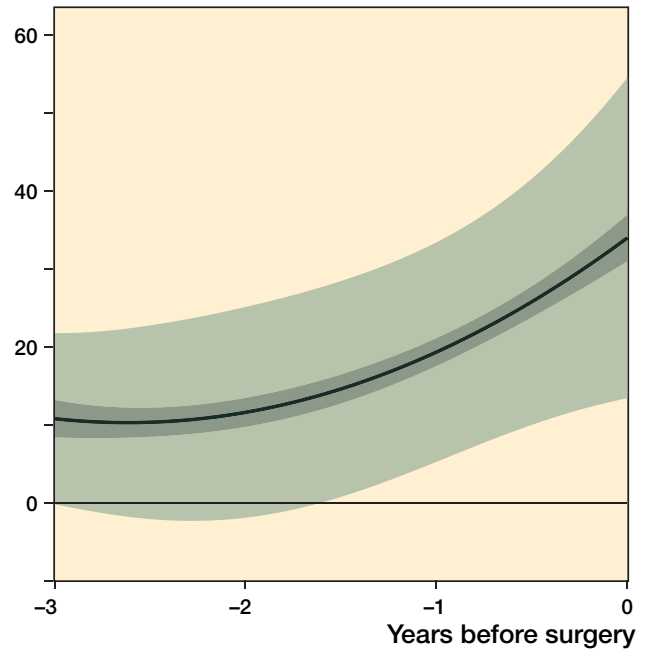

Figure 9. Model describing the development of population mean MP in in the 343 children who underwent preventive hip surgery. The model was adjusted for age at the time of MP measurement. $95 \% \mathrm{Cls}$ (dark) and $1 \mathrm{SD}$ of individual variation (light) are shown.

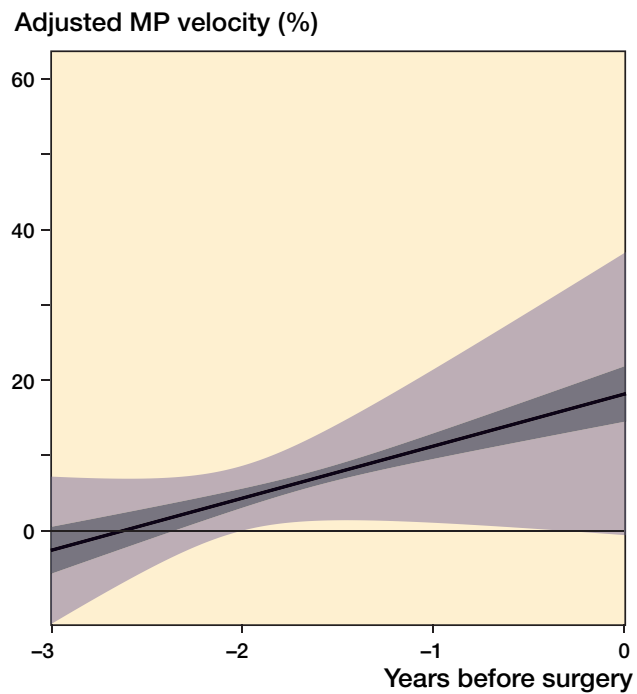

Figure 10. Model describing the development of population mean MP velocity in the 343 children who underwent preventive hip surgery. The model was adjusted for age at the time of MP measurement. 95\% Cls (dark) and 1 SD of individual variation (light) are shown. 